\title{
Self-Organization of the 2-Methoxyquinhydrone Charge Transfer Complex in Polar Planes
}

\author{
Marcel BOUVET*, Bernard MALEZIEUX, Patrick HERSON, Françoise VILLAIN \\ Laboratoire de Chimie Inorganique et Matériaux Moléculaires, Université Pierre et Marie Curie - Paris 6, CNRS - UMR 7071, 4 place Jussieu, Case \\ 5 courrier 42, 75252 Paris cedex 05, France.Fax: +331442738 41; Tel: +331442730 83; E-mail: marcel.bouvet@espci.fr
}

\begin{abstract}
Polar planes resulting from the association of polar stacks through hydrogen bonds, have been evidenced on single crystals of a substituted quinhydrone prepared by a one-pot oxidation-co${ }_{10}$ sublimation solvent-free process.
\end{abstract}

As a classical intermolecular complex example, the quinhydrone is widely used to illustrate how complexes can be built up around the subtle balance between non-covalent interactions, such as hydrogen bonding and $\pi-\pi$ stacking. ${ }^{1,2}$ In the quinhydrone, $\pi-\pi$ interactions ${ }_{15}$ exist inside the donor-acceptor stacks, whilst infinite molecular chains form through $\mathrm{H}$-bonds between neighboring stacks. ${ }^{3-5}$

Only few examples of resolved X-ray structures of quinhydrones built up from polar quinone or hydroquinone derivatives are available in the literature. One can mention those based on 1,420 naphthoquinone, ${ }^{6,7}$ phenanthrene-9,10-quinone, ${ }^{8}$ 1,4-dihydro- 9,10 anthraquinone ${ }^{9}$, triptycenequinone $\mathrm{e}^{10}$ and 2-methoxy-1,4benzoquinone. ${ }^{11}$ Nevertheless, molecules are packed generally in a head-to-tail configuration and dipole-dipole interactions are rarely discussed.

${ }_{25}$ Even though several quinhydrones can be carried out by simply grinding the starting materials in a mortar, ${ }^{12}$ the obtention of single crystals suitable for X-ray diffraction studies remains uneasy. To overcome the solvation energy effects involved in the crystallization process from solutions, an alternative synthesis carried out from the 30 solid forms, under vacuum, has been recently proposed. ${ }^{11}$ Nevertheless, this method was difficult to generalize, due to the difference of vaporization energies between the quinones and dihydroquinones.

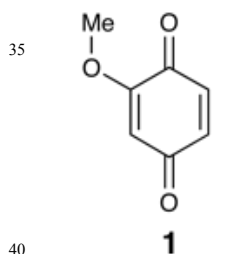

40

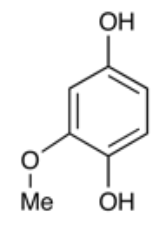

2

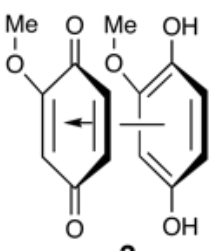

3
Scheme 1

Laboratoire de Chimie Inorganique et Matériaux Moléculaires, ${ }_{45}$ Université Pierre et Marie Curie - Paris 6, CNRS - UMR 7071, 4 place Jussieu, Case courrier 42, 75252 Paris cedex 05, France. Email: marcel.bouvet@espci.fr; Fax: +33 1442738 41; Tel: +33 144273083

$\dagger$ Electronic Supplementary Information (ESI) available: 1. Crystallographic data for compounds $\mathbf{2}$ and $\mathbf{3}$ are available free of charge from the Cambridge Data Center via Internet at http://www.ccdc.cam.ac.uk/data request/cif (2: CCDC 626643 and 3: 626640). 2. Raman spectrum of $\mathbf{3}$ compared to that of non-substituted quinhydrone.
Here, we report a novel approach, starting only from the substituted ${ }_{50}$ dihydroquinone. This route provides an in-situ generation of the oxidized counterpart (the corresponding quinone). That ensures a slow process particularly convenient for the single crystals' growth. The commercially available 2-methoxy-1,4-dihydroquinone $\mathbf{2}$, when heated in a sublimator under primary vacuum, in the presence, in a 55 separated container, of an organic or inorganic oxidizing agent (2,3,5,6-tetrachloro-1,4-benzoquinone or 2,3-dichloro-5,6-dicyano1,4-benzoquinone as well as $\mathrm{PbO}_{2}$ ), both at $50^{\circ} \mathrm{C}$, undergoes a redox reaction that leads to the formation of $1 . ¥$ Then, in situ, cosublimation of $\mathbf{1}$ and $\mathbf{2}$ leads, after few hours, to black prisms of $\mathbf{3}$ 60 (space group $\mathrm{P} 2{ }_{1} / \mathrm{c}$ ). $\S$ It is worth noting that, when the tetrasubstituted quinones were employed, the presence of tetrasubstituted moieties were not detected in any charge transfer complex. The ${ }^{1} \mathrm{H}$ NMR spectrum of crystals of $\mathbf{3}$ dissolved in $\mathrm{CD}_{3} \mathrm{CN}$ confirms that $\mathbf{1}$ and $\mathbf{2}$ are in a 1 to 1 ratio, with no shift ${ }_{65}$ compared to the isolated molecules. This is in agreement with the lack of charge transfer band in the absorption spectrum of $\mathbf{3}$ dissolved in acetonitrile, while the absorption spectrum of $\mathbf{3}$ obtained in the solid state from a $\mathrm{KBr}$ pellet, dark violet in color, shows a strong and broad absorption band centered at $560 \mathrm{~nm}$ (Fig. 1).

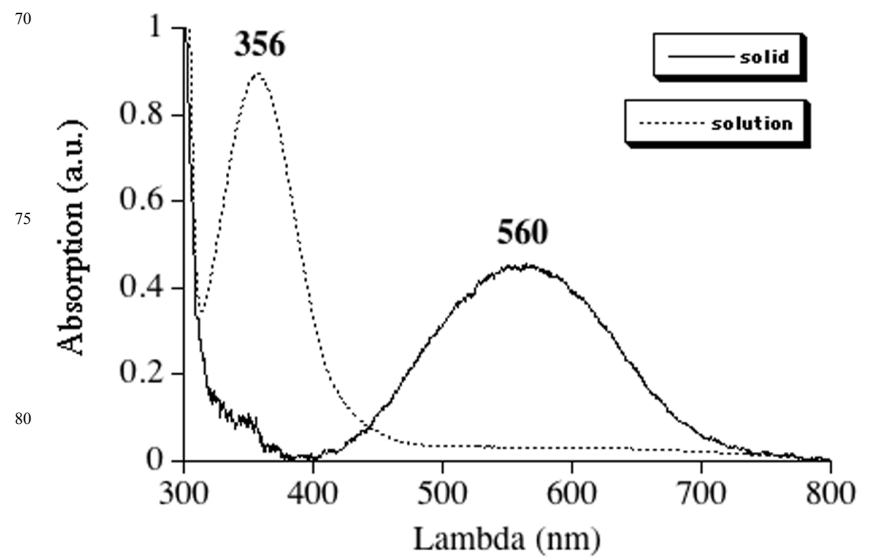

${ }_{85}$ Fig. 1 Optical absorption spectrum of $\mathbf{3}$ showing the charge transfer band (KBr pellet), compared to that of a 50:50 mixture of $\mathbf{1}$ and $\mathbf{2}$ (acetonitrile solution).

The Raman spectrum of single crystals of $\mathbf{3}$ exhibits very intense 90 bands compared to subunits 1 and $\mathbf{2}$ (Fig. 2). Due to the use of an incident laser wavelength belonging to the charge transfer absorption range, the experiment occurs in resonant Raman conditions. ${ }^{13,14}$ The main vibrations appear at 1671 and $1600 \mathrm{~cm}^{-1}$, attributed to the $8 \mathrm{a} \mathrm{C}$ $\mathrm{C}$ vibration of the benzene ring and to $\mathrm{C}=\mathrm{O}$ vibration, respectively, 95 both shifted by $20 \mathrm{~cm}^{-1}$ compared to the non-substituted quinhydrone. ${ }^{15}$ That confirms the existence of an intermolecular charge transfer complex. 


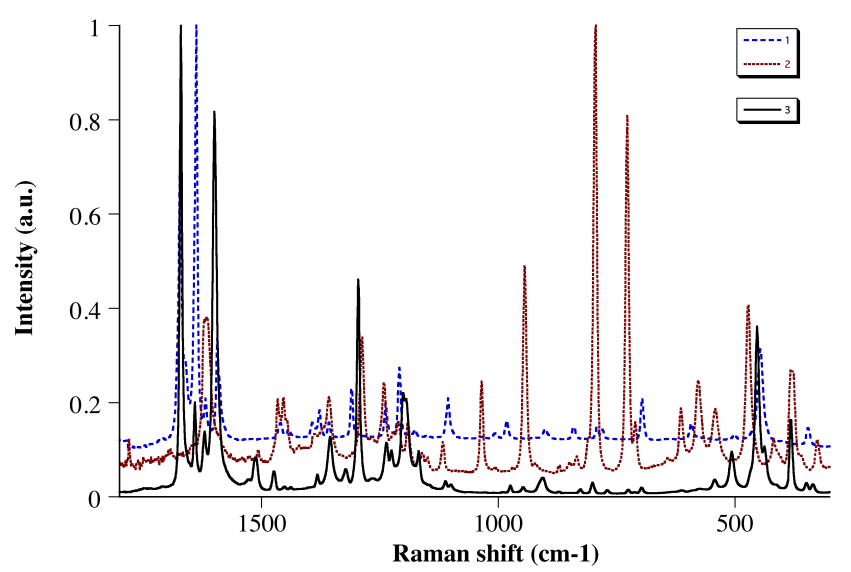

Fig.2 Raman spectrum of $\mathbf{3}$ (solid line) compared to that of the 100 quinone and hydroquinone subunits $\mathbf{1}$ and $\mathbf{2}$. All the spectra have been normalized.

The asymmetric unit is composed of one molecule of $\mathbf{1}$ and one molecule of 2 tilted by $4.7^{\circ}$ (Fig. 3a). As a matter of fact 3 is a 1:1

105 complex as the non-substituted quinhydrone. ${ }^{3-5}$ The molecules arrange in columns parallel to the a-axis and make an angle of 59.8 (subunit 1) and $55.2^{\circ}$ (subunit 2) with this axis (Fig. 3b). The shortest intermolecular distance between the mean planes is alternately 3.21 and $3.23 \AA$ all along the column axis, indicating 110 strong $\pi-\pi$ interactions.

The two C-OH bonds are slightly different, 1.372(7) and 1.387(7) $\AA$, for $\mathrm{C} 8 \mathrm{-O} 4$ and $\mathrm{C} 11-\mathrm{O} 8$, respectively, whereas the two carbonyl distances are equivalent, 1.234(7) $\AA$ for $\mathrm{C} 1-\mathrm{O} 1$ and $\mathrm{C} 4-\mathrm{O} 3$. In the quinone subunit, the $\mathrm{C}-\mathrm{C}$ bonds are 1.341(7) and 1.342(9) $\AA$ for the 115 double bonds, and between 1.461(9) and 1.507(8) $\AA$ for the single bonds; whereas in the hydroquinone subunit all the $\mathrm{C}-\mathrm{C}$ bonds lay in the range 1.385-1.401 $\AA$. Each molecule is linked to two neighbours belonging to adjacent columns via hydrogen bonds; dO3-O4' = $2.827(2) \AA\left(\mathrm{dO}_{3}-\mathrm{H} 4{ }^{\prime}=1.954(4) \AA\right)$, angle O3-H4'-O4' = $144^{\circ}$ and ${ }_{120} \mathrm{dO} 6^{\prime}-\mathrm{O} 1=2.802(2) \AA \AA$ (dH6'-O1 = 2.002(4) $\AA$ ), angle O6'-H6'-O1 $=146^{\circ}$. This provides a zig-zag chains' orientation defining sheets parallel to the (101) plane (Fig. 3b and 4). Additionaly, auxiliary $\mathrm{CH} . . . \mathrm{OCH}_{3}$ interactions participate to the intercolumnar cohesion (Fig 4), with dH51-O5 = 2.668(4) $\AA$ and dH101-O2 = 2.671(4) $\AA$.

125

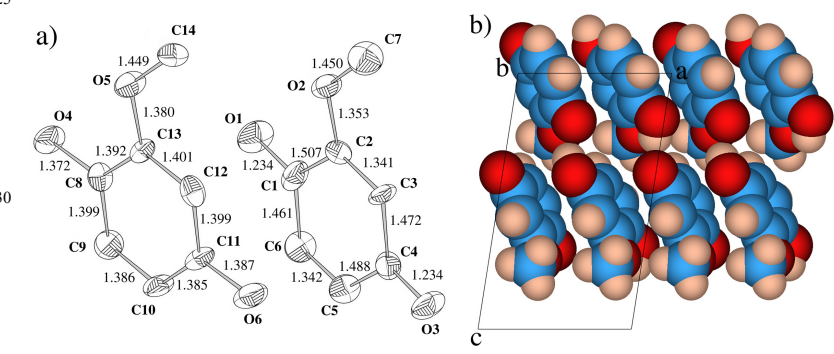

Fig. 3 X-ray crystal structure of 3: a) ORTEP representation (30\% probability level) of the asymmetric unit including atomic numbering and bond lengths in $\AA$; b) view of molecules in two adjacent columns connected via $\mathrm{H}$-bonds ( $80 \%$ van der Waals radii).

${ }_{140}$ Considering the structural pattern obtained from single crystal diffraction studies, two main features have caught our attention: Firstly, the quinhydrone $\mathbf{3}$, formed by the alternate stack of molecules $\mathbf{1}$ and $\mathbf{2}$, exhibits an exact superimposition of the methoxy substituents (Fig. 3b). Secondly, molecules belonging to H-bonded 145 chains are tilted by $44.7^{\circ}$ with respect to each other as defined by the torsion angle between $\mathrm{C} 1$ to $\mathrm{C} 6$ and $\mathrm{C} 8$ to $\mathrm{C} 13$ planes, whereas those in next sheet are generated by the inversion center. Thus, the dipolar character of subunits $\mathbf{1}$ and $\mathbf{2}$ due to their methoxy arm is preserved in a column and on the scale of a plane. The planes ${ }_{150}$ correspond to each other by an inversion center. It is worth noting that in pure $1,{ }^{16}$ molecules lie in a head-to-tail arrangement, with an intersheet distance of $3.3 \AA$, whereas in pure $\mathbf{2}$ obtained as single crystal by sublimation under vacuum (this work) $\rrbracket, \pi-\pi$ interactions do not occur, and crystal arrangement results from strong $\mathrm{H}$-bonds 155 providing a pattern made of perpendicular interlocked molecules.

In order to appreciate their role in the building up, the dipole moments of $\mathbf{1}$ and $\mathbf{2}$ were calculated by Chem3D Pro ${ }^{\mathrm{TM}}$ software with the $\mathrm{MNDO}^{17}$ method using the geometrical data collected from single crystals of $\mathbf{3}$ without geometrical optimization. In $\mathbf{1}$ the dipole 160 moment, oriented parallel to the $\mathrm{C} 1-\mathrm{O} 2$ direction, is 1.0 Debye, compared to $0.8 \mathrm{D}$ for the optimized geometry. ${ }^{16}$ In 2 , it is 1.5 Debye and is turned to the $\mathrm{C} 8-\mathrm{C} 12$ direction. So, in the structure, although the dipole moments of $\mathbf{1}$ and $\mathbf{2}$ remain not aligned, the resulting dipole moment for the corresponding quinhydrone pair appears to be ${ }_{165} 2.0$ Debye, as calculated for a A-D pair, whose projection in the $\left(\begin{array}{c}1 \\ \mathrm{~b} \cdot \mathrm{c}\end{array}\right)$ plane points in the $\mathrm{C} 1-\mathrm{C} 2$ direction (Fig. $4 \mathrm{a}$ ).
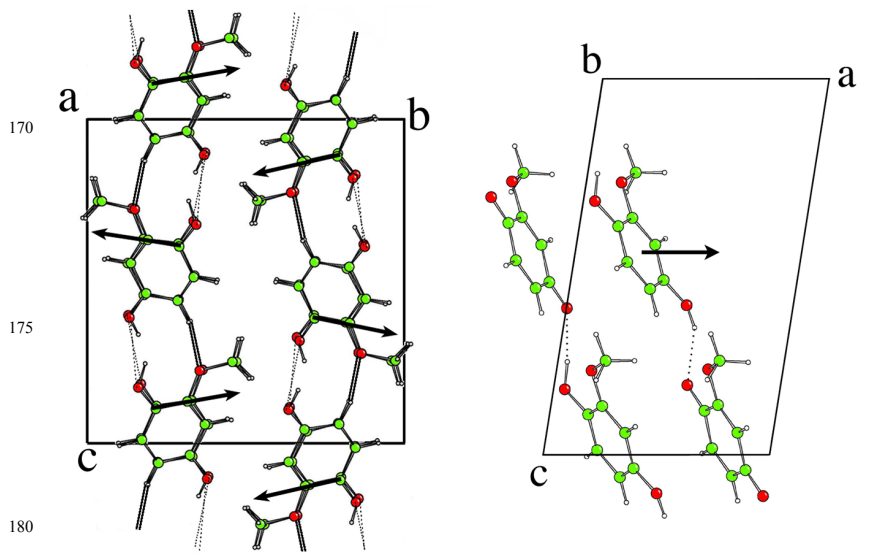

Fig. 4 X-ray crystal structure of 3: projection on the plane (011) (left), Hbonds are indicated as dotted lines, and $\mathrm{CH}$... O interactions as full lines; arrows indicate the projection of the dipole moment for each column; 185 projection on the plane (101) (right), arrow indicate the orientation of the resulting dipole moment for a plane.

Calculations on two A-D pairs associated by two H-bonds indicate that the resulting dipole moment (3.0 Debye) is oriented mainly 190 parallel to the $\left(\begin{array}{l}1 \\ \mathrm{a} \cdot \mathrm{c}\end{array} \mathrm{c}\right)$ plane (Fig. 4), with only a minor component in the $\left(\begin{array}{l}r \\ b \cdot c\end{array}\right)$ plane. As a consequence of the columns' association provided by the hydrogen bonds, one can conclude that real polar planes are obtained. This unusual 2D polar arrangement associated with the presence of a charge transfer band opens the way to new 195 materials (polar films are envisaged) that should exhibit particular electrooptical properties.

This work proposes an efficient one-pot solvent-free method to make crystals of substituted quinhydrones, starting from 200 dihydroquinones. 
We would like to thank the E.S.P.C.I - Paris for supporting this work. O. Ben Youchrett-Zallez (FSB - Bizerte (Tunisia) and UPMC) is gratefully acknowledged for cyclic voltammetry experiments.

\section{Notes and references}

205 \$The same product was obtained from $\mathbf{1}$ and $\mathbf{2}$, in an equimolar ratio, by heating 1 at $50{ }^{\circ} \mathrm{C}$ while 2 was kept at room temperature, in a separated compartment of a home-made sublimator. For this purpose, $\mathbf{1}$ was synthesized from 2 by oxidization at room temperature with 1,4benzoquinone. ${ }^{11}$ This reaction can be explained from the redox potentials

210 of $\mathbf{1}$ and $\mathbf{2}$ that we determined by cyclic voltammetry in acetonitrile, as well as these of the unsubstituted analogs. 1 shows two monoelectronic and reversible reduction waves at $-0.94 \mathrm{~V}$ and $-1.75 \mathrm{~V}$ versus ferricinium/ferrocene $\left(\mathrm{Fc}^{+} / \mathrm{Fc}\right)$, while 2 shows one irreversible oxidation wave at $+0.56 \mathrm{~V} \mathrm{vs} \mathrm{Fc}^{+} / \mathrm{Fc}$. Due to the presence of the donating methoxy

215 group, $\mathbf{1}$ is more difficult to reduce than the 1,4-benzoquinone by $0.1 \mathrm{~V}$, and $\mathbf{2}$ is easier to oxidize than the non substituted 1,4-hydroquinone, also by $0.1 \mathrm{~V}$.

$¥$ Crystals of 3 were synthesized from 2 (15 mg, $0.14 \mathrm{mmol})$ and 2,3,5,6tetrachloro-1,4-benzoquinone $(20 \mathrm{mg}, 0.08 \mathrm{mmol})$ or 2,3-dichloro-5,6-

220 dicyano-1,4-benzoquinone $(20 \mathrm{mg}, 0.09 \mathrm{mmol})$ in two separated compartments of an home-made sublimator, both heated under vacuum $(10$ ${ }^{1}$ Torr) at $50{ }^{\circ}$ C. 3: M.P.: $103^{\circ} \mathrm{C}(\mathrm{dec}$ ), IR (KBr): 1676 (s), 1648 (m) (C=O) $\mathrm{cm}^{-1}$, Raman $(785 \mathrm{~nm}$ beam of a laser diode used in a microRaman set-up on a single crystal): $1671(8 \mathrm{a})^{15}, 1600(\mathrm{C}=\mathrm{O}), 453$ (A band) $\mathrm{cm}^{-1}$, UV-

${ }_{225}$ Visible (KBr pellet): $\lambda_{\max } 560 \mathrm{~nm} .{ }^{1} \mathrm{H}$ NMR of dissolved crystals (300 $\left.\mathrm{MHz}, \mathrm{CD}_{3} \mathrm{CN}\right): \delta=3.81\left[\mathrm{~d}, \mathrm{~J}_{\mathrm{OCH} 3-5}=0.4 \mathrm{~Hz}, 3 \mathrm{H}, \mathrm{OCH}_{3}(\mathbf{1})\right] ; 3.82[\mathrm{~s}, 3 \mathrm{H}$, $\mathrm{OCH}_{3}(2)$ ]; 5.93 [s broad, $1 \mathrm{H}, \mathrm{OH}(2)$ ], 6.01 [ddq, $\mathrm{J}_{5-6}=1.9 \mathrm{~Hz}, \mathrm{~J}_{5-3}=0.4$ $\left.\mathrm{Hz}, \mathrm{J}_{5-\mathrm{OCH} 3}=0.4 \mathrm{~Hz}, 2 \mathrm{H}, \mathrm{H}_{5}(\mathbf{1})\right], 6.27\left[\mathrm{dd}, \mathrm{J}_{5-6}=8.5 \mathrm{~Hz}, \mathrm{~J}_{5-3}=2.8 \mathrm{~Hz}, 1 \mathrm{H}\right.$, $\left.\mathrm{H}_{5}(2)\right] ; 6.42$ [s broad, $\left.1 \mathrm{H}, \mathrm{OH}(2)\right], 6.47\left[\mathrm{~d}, \mathrm{~J}_{5-3}=2.8 \mathrm{~Hz}, 1 \mathrm{H}, \mathrm{H}_{3}(2)\right] ; 6.66$ $230\left[\mathrm{~d}, \mathrm{~J}_{5-6}=8.5 \mathrm{~Hz}, 1 \mathrm{H}, \mathrm{H}_{6}(\mathbf{2})\right], 6.705\left[\mathrm{~d}, \mathrm{~J}_{5-6}=1.9 \mathrm{~Hz}, 2 \mathrm{H}, \mathrm{H}_{6}(\mathbf{1})\right], 6.71\left[\mathrm{~d}, \mathrm{~J}_{3-}\right.$ $\left.{ }_{5}=0.4 \mathrm{~Hz}, 2 \mathrm{H}, \mathrm{H}_{3}(\mathbf{1})\right]$. Anal. Calcd for $\mathrm{C}_{14} \mathrm{H}_{14} \mathrm{O}_{6}$ : C, $60.43 \%$; H, 5.07\%. Found: C, $60.07 \%$; H, $5.23 \%$.

$\S$ Crystal data for 3: $\mathrm{C}_{14} \mathrm{H}_{14} \mathrm{O}_{6}, \mathrm{Fw}=278.26$, black prismatic crystal $(0.08$ x $0.12 \times 0.14 \mathrm{~mm})$, monoclinic, $\mathrm{P} 2_{1} / \mathrm{c}, \mathrm{a}=7.8386(10), \mathrm{b}=12.791(3), \mathrm{c}=$ 23513.187 (2) $\AA, \alpha=90, \beta=99.170$ (11), $\gamma=90^{\circ}, V=1305.3$ (4) $\AA^{3}, Z=4$, $D_{c}=1.42 \mathrm{~g} \mathrm{~cm}^{-3}$, were measured on a KAPPACCD - Nonius diffractometer. $\lambda(\mathrm{MoK} \alpha)=0.71073 \AA, \mu(\mathrm{MoK} \alpha)=1.14 \mathrm{~cm}^{-1}, 11762$ reflections $\left(\mathrm{T}=200 \mathrm{~K}, 2<\theta<30^{\circ}\right)$, nb of independent data collected: 3773 , $\mathrm{nb}$ of independent data used for refinement: $1213(\mathrm{Fo})^{2}>1.5 \sigma(\mathrm{Fo})^{2}$,

240 merging $\mathrm{R}=0.039, \mathrm{R}=\Sigma|| \mathrm{Fo}|-| \mathrm{Fc} \| / \Sigma \mid \mathrm{Fo}=0.0675, \mathrm{Rw}^{*}=[\Sigma \mathrm{w}(\| \mathrm{Fo} \mid$ - $\left.|\mathrm{Fc}| \mid)^{2} / \Sigma \mathrm{wFo}^{2}\right]^{1 / 2}=0.0570,-0.41<\Delta \rho<0.33$.

q Crystal data for 2: $\mathrm{C}_{6} \mathrm{H}_{8} \mathrm{O}_{3}, \mathrm{Fw}=140.14$, colorless parallelepiped crystal (0.06 × $0.10 \times 0.12 \mathrm{~mm})$, monoclinic, $\mathrm{P} 2_{1} / \mathrm{n}, \mathrm{a}=4.9678(7), \mathrm{b}=10.533(4)$, $\mathrm{c}=13.094(2) \AA, \alpha=90, \beta=91.194(13), \gamma=90^{\circ}, \mathrm{V}=685.0(3) \AA^{3}, Z=4$,

$245 \mathrm{D}_{\mathrm{c}}=1.359 \mathrm{~g} \mathrm{~cm}^{-3}, 3578$ reflections $\left(\mathrm{T}=295 \mathrm{~K}, 3<\theta<27.5^{\circ}\right)$, nb of independent data collected: 1564, nb of independent data used for refinement: $978(\mathrm{Fo})^{2}>1.5 \sigma(\mathrm{Fo})^{2}$, merging $\mathrm{R}=0.047, \mathrm{R}=\Sigma\|\mathrm{Fo}|-| \mathrm{Fc}\|$ $/ \Sigma \mid \mathrm{Fo}=0.0473, \mathrm{RW}^{*}=\left[\Sigma \mathrm{w}(\|\mathrm{Fo}|-| \mathrm{Fc}\|)^{2} / \Sigma \mathrm{wFo}^{2}\right]^{1 / 2}=0.0595$, $0.28<\Delta \rho<0.25$.

250

1 G. R. Desiraju, Crystal Engineering: The design of Organic Solids, Elsevier, Amsterdam, 1989.

2 D. Braga, F. Grepioni and G. R. Desiraju, Chem. Rev. 1998, 98, 13751405.

2553 H. Matsuda, K. Osaki and I. Nitta, Bull. Chem. Soc. Jpn. 1958, 31, 611.

4 T. Sakurai, Acta Crystallogr. 1965, B19, 320.

5 T. Sakurai, Acta Crystallogr. 1968, B24, 403-412.

6 A. Thozet and J. Gaultier, Acta Crystallogr. 1977, B33, 1052-1057.

7 A. Artiga, J. Gaultier, C. Hauw and C. Nguyen Ba, Acta Crystallogr.

260 1978, B34, 1212-1218.

8 F. Calderazzo, C. Forte, M. Fabio, G. Pampaloni and L. Pieretti, Helv. Chim. Acta 2004, 87, 781-789.
9 B. S. Joshi, T. K. Rho, P. L. Rinaldi, W. X. Liu, T. A. Wagler, M. G. Newton, D. W. Lee and S. W. Pelletier, J. Chem. Crystallogr. 1997, 27, $265 \quad 553-559$.

10 K. Yamamura, T. Kawashima, K. Eda, F. Tajima and M. Hashimoto, J. Mol. Struct. 2005, 737, 1-6.

11 M. Bouvet, B. Malézieux and P. Herson, Chem. Commun. 2006, 17511753.

27012 A. O. Patil, D. Y. Curtin and I. C. Paul, J. Am. Chem. Soc. 1984, 106, 348-353.

13 S. Sugai and G. Sato, Solid State Commun. 1986, 58, 759-763.

14 A. M. Moran and A. M. Kelley, J. Chem. Phys. 2001, 115, 912-924.

15 Y. Uchida, C. Okabe, A. Kishi, H. Takeshita, Y. Suzuki, Y. Nibu, R.

275 Shimada and H. Shimada, Bull. Chem. Soc. Jpn. 2002, 75, 695-703.

16 E. M. D. Keegstra, A. L. Spek, J. W. Zwikker and L. W. Jenneskens, J. Chem. Soc., Chem. Commun. 1994, 1633-1634.

17 M. J. S. Dewar and M. Thiel, J. Am. Chem. Soc. 1977, 99, 4899-4907. 